\title{
REMEDIACIÓN DE UN SUELO DE LA CUENCA ALTA DEL RÍO BOGOTÁ CONTAMINADO CON LOS METALES PESADOS CADMIO Y CROMO
}

\section{REMEDIATION OF A SOIL CONTAMINATED WITH THE HEAVY METALS CADMIUM AND CROMIUM ON THE HIGH BASIN OF THE BOGOTA RIVER}

\author{
Rodrigo Lora Silva'; Helver Bonilla Gutiérrez²
}

${ }^{1}$ Ing. Químico, M.Sc. Facultad de Ingeniería Agronómica, Universidad de Ciencias Aplicadas y Ambientales, U.D.C.A. rodrigolorasilva@yahoo.com ${ }^{2}$ Químico, Universidad de Ciencias Aplicadas y Ambientales, U.D.C.A. hbonilla@udca.edu.co

Rev. U.D.C.A Act. \& Div. Cient. 13 (2): 61-70, 2010

RESUMEN

Suelos contaminados con metales pesados tóxicos, como cadmio y cromo, originan riesgo para los animales y para los humanos, que consumen productos, que en ellos se producen. Se hace necesaria la remediación de estos suelos, por lo cual, en un trayecto entre Villapinzón y Bosa (Cundinamarca), se analizaron muestras de suelo de $0-20 \mathrm{~cm}$ de profundidad, que mostraron un contenido de $\mathrm{Cd}$ y $\mathrm{Cr}$, medio a alto, indicando contaminación. Con uno de ellos, cercano al Río Bogotá, se produjo un bioensayo, para conocer el efecto de remediación bajo condiciones de casa de malla, empleando lechuga y pasto ryegrass. Las fuentes y las dosis de los materiales remediados fueron diferentes dosis de $\mathrm{CaSO}_{4}$, de $\mathrm{CaCO}_{3}$, de $\mathrm{Ca}\left(\mathrm{H}_{2} \mathrm{PO}_{4}\right)_{2}$, de $\mathrm{FeSO}_{4}$ y de diatomácea activada. El diseño empleado fue bloques completamente al azar, con tres replicaciones y un testigo, para cada una de las especies vegetales manejadas. Los resultados mostraron que el contenido de $\mathrm{Cd}$ y de $\mathrm{Cr}$ de lechuga y de pasto era elevado en el testigo. Para ryegrass, la aplicación de $6000 \mathrm{~kg} \mathrm{ha}^{-1}$ de $\mathrm{CaCO}_{3}$ o de $600 \mathrm{~kg} \mathrm{ha}^{-1}$ de $\mathrm{Ca}\left(\mathrm{H}_{2} \mathrm{PO}_{4}\right)_{2}$ redujo el $\mathrm{Cd}$, a niveles no tóxicos; para $\mathrm{Cr}$, la aplicación de $4000 \mathrm{~kg} \mathrm{ha}^{-1}$ de $\mathrm{CaCO}_{3}$ o de $400 \mathrm{~kg} \mathrm{ha}^{-1}$ de $\mathrm{FeSO}_{4}$ rebajó, significativamente, su contenido. Para lechuga, la aplicación de $6000 \mathrm{~kg} \mathrm{ha}^{-1}$ de $\mathrm{CaCO}_{3}$ o de $2000 \mathrm{~kg} \mathrm{ha}^{-1}$ de diatomácea activada disminuyó, representativamente, el Cd en la planta. Para $\mathrm{Cr}$, el $\mathrm{CaCO}_{3}$ o el $\mathrm{FeSO}_{4}$ a niveles de $2000 \mathrm{~kg} \mathrm{ha}^{-1}$ y $400 \mathrm{~kg} \mathrm{ha}^{-1}$, respectivamente, redujeron significativamente su contenido en la planta.

Palabras clave: Remediación, contaminación, metales tóxicos, cadmio, cromo.

\section{SUMMARY}

Soil contaminated with heavy toxic metals such as $\mathrm{Cd}$ o $\mathrm{Cr}$ produce risks to animals and humans that consume the products produced in it. It is, therefore necessary to remedy these soils. Soil samples at $0-20 \mathrm{~cm}$ depths were taken in a catena from Villapinzon to Bosa (Cundinamarca) at the high basin of the Bogota river. The available content of $\mathrm{Cd}$ and $\mathrm{Cr}$ was medium to high indicating contamination problems in these soils. In a soil taken near the river at the Bosa village, under screen house conditions a bio-essay was carried out to establish the effect of some materials using as indicator plants lettuce and ryegrass. The sources were $\mathrm{CaSO}_{4}, \mathrm{CaCO}_{3}$, $\mathrm{Ca}\left(\mathrm{H}_{2} \mathrm{PO}_{4}\right)_{2}, \mathrm{FeSO}_{4}$ and activated diatomaceous at different levels. A complete randomized block design with three replicates and a check was used for each indicator plant. The results showed that the $\mathrm{Cd}$ and $\mathrm{Cr}$ content in the check were high in both indicator plants. The application of $6000 \mathrm{~kg} \mathrm{ha}^{-1}$ of $\mathrm{CaCO}_{3}$ or $600 \mathrm{~kg} \mathrm{ha}^{-1}$ of $\mathrm{Ca}\left(\mathrm{H}_{2} \mathrm{PO}_{4}\right)_{2}$ on ryegrass reduced the $\mathrm{Cd}$ to non toxic level. The application of $4000 \mathrm{~kg} \mathrm{ha}^{-1}$ of $\mathrm{CaCO}_{3}$ or $400 \mathrm{~kg} \mathrm{ha}^{-1}$ of $\mathrm{FeSO}_{4}$ reduced significatively the $\mathrm{Cr}$ content. In lettuce the application of $6000 \mathrm{~kg} \mathrm{ha}^{-1}$ of $\mathrm{CaCO}_{3}$ or $2000 \mathrm{~kg} \mathrm{ha}^{-1}$ of activated diatomacea reduced the $\mathrm{Cd}$ concentration to non toxic level. The application of $2000 \mathrm{~kg}$ ha-1 of $\mathrm{CaCO}_{3}$ or $400 \mathrm{~kg} \mathrm{ha}^{-1}$ of $\mathrm{FeSO}_{4}$ reduced significantly the $\mathrm{Cd}$ concentration.

Key words: Remediation, contamination; toxic metals, cadmium, chromium. 


\section{INTRODUCCIÓN}

En Colombia existen zonas cuyos suelos, a través del tiempo, se han estado contaminando con metales pesados tóxicos, que sin ser esenciales para las plantas, son tóxicos para el hombre y para los animales que los ingieran, cuando se sobrepasan determinados niveles. Entre estos elementos se registran el cadmio $(\mathrm{Cd})$, el cromo $(\mathrm{Cr})$, el mercurio $(\mathrm{Hg})$, el plomo $(\mathrm{Pb})$, el arsénico (As) y el selenio (Se) (Lora, 2007; Lora, 1996). La contaminación de suelos y de aguas por estos metales, se deriva de la explotación y la fundición de metales, de los insumos agrícolas, de lodos y de sedimentos residuales, de la combustión del carbón y del petróleo, industrias químicas y de la inadecuada disposición de desechos urbanos e industriales (Siebe, 1994; Orozco et al. 2005; Muchuweti et al. 2006). Entre los factores que afectan el comportamiento de los metales citados en los suelos, están: pH del suelo, materia orgánica, contenido y tipo de arcillas, óxidos del hierro, manganeso y aluminio, carbonatos y condiciones de óxido-reducción (Matamoros, 2003). Algunos efectos derivados del impacto biológico, son: el cadmio es tóxico y carcinógeno; el cromo hexavalente es altamente tóxico y carcinógeno; el mercurio es teratogénico, es decir, que produce efectos congénitos; el plomo es altamente tóxico y el arsénico es igualmente tóxico y teratogénico. En Colombia, las causas más frecuentes de contaminación por metales pesados tóxicos de suelos y los cultivos, han sido la adición masiva de insumos agrícolas y de lodos de albañal, como práctica fertilizante; la deposición de elementos y de sustancias atmosféricas generadas por la industria; los motores de combustión interna; la lluvia ácida; el derrame de crudos y de residuos de minerías; el riego con aguas contaminadas y las descargas de aguas negras, y la deposición de sedimentos seleníferos y molibdeníferos (Matamoros, 2003; Mejía, 1998; Álvarez et al. 2006; Cuberos et al. 2009).

Estudios adelantados acerca del río Bogotá, han mostrado la presencia de elementos tóxicos, como $\mathrm{Hg}, \mathrm{Pb}, \mathrm{Cd}, \mathrm{Cr}$, As, entre otros y debido a que en algunos casos el río se emplea en riego, existe un proceso activo de contaminación de suelos, de plantas y de animales, hasta niveles no permisibles, para humanos y para animales (Alfaro et al. 2002). A nivel de Colombia, los trabajos de remediación son muy escasos (Mejía E Osorio, citados por Lora, 1996; Mejía, 2002).

La cuenca alta del río Bogotá está situada en la posición de Vega (plano de inundación), formando fajas estrechas de gran longitud, lo mismo que a lo largo de sus afluentes. Se extiende, en el norte, desde el Municipio de Villapinzón hasta el páramo de Sumapaz al sur, en las inmediaciones del Salto de Tequendama. Algunos de estos suelos están sujetos a inundaciones, por lo cual, se requiere obras para evitarlas y así desarrollar agricultura y ganadería sin problemas. La topografía es plana, con pendientes dominantes de 0 a $1 \%$, lo que hace que el drenaje externo sea deficiente. En general, son suelos ácidos, franco a franco arcilloso y fertilidad media. La extensión es de 29.377ha equivalente al 20\% del área total de la sabana de Bogotá (López, 1976).

Naturalmente, el cadmio está asociado a minerales de zinc y de fósforo, a manera de impureza, por lo que los fertilizantes basados en estos elementos pueden contener cadmio, que se llega a convertir en contaminante y acumularse, posteriormente, en los organismos vivos que ingieran material vegetal, procedente de suelos, a los que se les ha aplicado, continuamente, zinc y fósforo (Lora, 2007; Bonomelli et al. 2003). En las plantas, la absorción de cadmio es determinada, principalmente, por su concentración en el suelo, por el $\mathrm{pH}$ $\mathrm{y}$ las especies vegetales en estudio, donde se acumula, de manera preferente, en las hojas, con cantidades que oscilan entre 0,07 y 18 ppm (Peterson \& Girling, 1981). En los suelos ácidos, el elemento es más disponible para las plantas. Aun en concentraciones bajas, el cadmio es persistentemente tóxico y se centraliza en cadenas alimenticias. Las plantas no poseen mecanismos de excreción para el metal (Badillo \& Pomades, 1995; Álvarez et al. 2008).

Laredo \& Cuesta (1987) reportan que las concentraciones de cadmio en especies forrajeras de diferentes regiones de Colombia oscilan entre 0,01 y 0,84ppm; para ganado vacuno, con contenidos superiores a 0,23ppm, se puede presentar toxicidad en los animales.

El cromo, se halla en rocas, en el suelo, en animales y en las plantas, en concentraciones variables. Presenta valencia tres (III) y seis (VI). El cromo trivalente es esencial para los seres humanos, en los que promueve la acción de la insulina. Los derivados del cromo hexavalentes (cromatos y dicromatos), usualmente, son de origen antropogénico, que en sus altas concentraciones producen toxicidad para las plantas, ocasionando disminución en la incorporación de calcio, de potasio, de fósforo, de hierro y de manganeso, además, de afecciones en el metabolismo de los carbohidratos y disminución de la clorofila. Algunos factores que afectan su disponibilidad son el $\mathrm{pH}$ del suelo, la interacción con otros elementos y compuestos orgánicos quelados (Peterson $\varepsilon$ Girling, 1981; Panda E Choudhury, 2005; Conceicão et al. 2007; Cuberos et al. 2009). Se utiliza en metalurgia, en curtido de cueros y en fabricación de pinturas; los efluentes de cromo hexavalente de estas industrias contaminan el agua y el suelo.

En el suelo, los metales pesados pueden quedar retenidos por procesos de adsorción, de complejación y de precipitación, ser absorbidos por las plantas y así incorporarse a las cadenas tróficas. También pueden pasar a la atmosfera por volatilización y movilizarse a las aguas superficiales o subterráneas (García E Dorronsoro, 2005; Paim et al. 2006). 
Como algunas estrategias empleadas en la disminución de metales pesados tóxicos, como cadmio y cromo, en suelos agrícolas, se consideran: a) la incorporación de materia orgánica, por su capacidad para formar quelatos estables; b) el incremento de $\mathrm{pH}$, mediante cales, óxidos e hidróxido de calcio, hasta pH de 6-7, con lo cual, los metales pesados pierden la capacidad de ser absorbidos por las plantas, por estar insolubles a pH superior a 6,0 (Lora, 2007; Marschner, 2003); c) el aporte de fosfatos, debido a la formación de sales insolubles con los metales pesados (Mejía, 1998; Matamoros, 2003) y d) el aporte de materiales silicatados, tanto por la formación de complejos como por el efecto indirecto sobre el $\mathrm{pH}$, puesto que el anión $\mathrm{SiO}_{3}{ }^{=}$en disociación en el agua del suelo, funciona como una base Bronsfed-Lowy, capturantes de iones $\mathrm{H}^{+}$(Méndez et al. 2000; Lora, 2001; Mejía, 2002; Kede et al. 2008; Accioly et al. 2009). Adicionalmente, puede haber remediación microbial, en especial, de suelos contaminados con cadmio (Sinha E Kumar, 2009).

La lechuga Romana (Lactuca sativa) y el pasto ryegrass anual (Lolium multiflorum) son especies vegetales sembradas en la zona bajo estudio. La lechuga es una de las principales hortalizas cultivadas en Colombia, por su volumen de consumo. Se producen desde el nivel del mar, en la Costa Atlántica, hasta a los $2.600 \mathrm{msnm}$, como en la Sabana de Bogotá. Es una plantación de fácil manejo (Osorio $\mathcal{E}$ Lobo, 1977; Lora et al. 2006).

El ryegrass anual conocido como ryegrass italiano, es un pasto amacollado anual de clima fresco. El sabor agradable y alta digestibilidad hacen de esta especie un forraje con alto valor para la alimentación del ganado. El mejor crecimiento ocurre cuando el pH del suelo es 5,5 a 7,5 y fertilidad media a alta del suelo.

Comúnmente, se destina para pastoreo en mezcla con tréboles, pero se puede usar para corte, heno y ensilaje. En mezcla con alfalfa, en algunas zonas de Colombia, produce un excelente forraje de corte o ensilaje, para la producción de leche (Estrada, 2002).

Las diatomáceas son algas microscópicas con caparazón silíceo. Cuando la célula muere, el contenido orgánico se destruye y el esqueleto de sílice pasa, generalmente, al fondo del agua, para formar, al cabo del tiempo, grandes depósitos, conocidos como tierra diatomácea, el cual, es un material inerte no tóxico. Sometiendo el material a $200^{\circ} \mathrm{C}$ durante una hora y posterior molienda a 200 mallas, se obtiene la diatomácea activada. Algunos trabajos han mostrado que la diatomácea activada, al igual que el silicato de calcio, pueden reducir la disponibilidad para la planta de algunos metales pesados tóxicos (Datnoff et al. 2001; Lora et al. 2002; Accioly et al. 2009).

\section{MATERIALES Y MÉTODOS}

La investigación, se realizó en la Universidad de Ciencias Aplicadas y Ambientales U.D.C.A, a $2.570 \mathrm{msnm}$ y temperatura promedio de $14^{\circ} \mathrm{C}$, bajo condiciones de casa de malla. Como plantas indicadoras, se adoptó la lechuga romana y el pasto ryegrass, que se comportan bien bajo casa de malla, además que son buenas indicadoras para la absorción de metales pesados y son representativas de hortalizas y de pastos utilizados en la zona bajo estudio. Se tomaron muestras de 0 a $20 \mathrm{~cm}$ de profundidad, en un trayecto entre el municipio de Villapinzón y el municipio de Bosa, en Cundinamarca y localizadas, desde el borde del río Bogotá, así: Villapinzón $\left(M_{1}\right)$, a $200 \mathrm{~m}$ del pueblo, junto al río; Guaymaral: $\left(M_{2}\right)$, a 300m, $\left(M_{3}\right)$, a $150 \mathrm{~m}$ y $\left(M_{4}\right)$, junto al río; Cota $\left(M_{5}\right)$, a $100 \mathrm{~m}$ del río; Florida $\left(M_{6}\right)$, a $100 \mathrm{~m}$; Bosa: $\left(M_{7}\right)$, a $300 \mathrm{~m}$ del río en potrero, $\left(M_{8}\right)$, a $100 \mathrm{~m}$ del río y $\left(M_{g}\right)$, sedimento río Bogotá.

Los metales bajo estudio, se extrajeron con solución $0,5 \mathrm{~N}$ de $\mathrm{HCl}$, considerada apropiada para extraer la fracción disponible (Mejía, 1998). En la tabla 1, se muestra el análisis de suelos para cadmio y para cromo.

Del sitio $\left(M_{8}\right)$, se tomaron $250 \mathrm{~kg}$ de $0-20 \mathrm{~cm}$ de profundidad, por tener un contenido medio a alto de cadmio y de cromo y por estar localizada en una finca productora de leche y de hortalizas, en la que se puede hacer, posteriormente, la investigación bajo condiciones de campo. El suelo, se secó y se molió en la casa de malla de la U.D.C.A, para hacer el bioensayo. El sitio muestreado corresponde a la asociación Aeric Epiaquents Fluvaquentic Endoaquepts, de símbolo RMO, con drenaje pobre a muy pobre.

Algunas características del suelo manejado en el bioensayo, son: $\mathrm{pH} 4,88$, densidad aparente $1,38 \mathrm{~g} / \mathrm{cm}^{3}$, conductividad eléctrica $1,12 \mathrm{mmhos} / \mathrm{cm}$, materia orgánica $2,96 \%$, textura franco-limoso, alófana negativo y aluminio intercambiable $3,00 \mathrm{me} / 100 \mathrm{~g}$.

Para los experimentos, se empleó un diseño de bloques completamente al azar, para cada especie vegetal, con tres repeticiones, constituidas por materas plásticas, con $2.000 \mathrm{~g}$ de suelo seco y molido, a tamaño de $2 \mathrm{~mm}$. En la tabla 2 aparecen los tratamientos aplicados.

Las fuentes remediadoras, se incorporaron con el suelo; se les aplicó agua, para mantenerlo a capacidad de campo (50\%) y se dejó reaccionar durante diez días, al cabo de los cuales, se trasplantaron plántulas de lechuga y se sembraron semillas de ryegrass. Se suministraron dos riegos semanales y al cabo de 60 días de siembra, se procedió a la cosecha del material vegetal y se determinó el rendimiento en base húmeda y seca, a $70^{\circ} \mathrm{C}$. 
Tabla 1. Contenido de $\mathrm{Cd}$ y $\mathrm{Cr}$ en las muestras de suelo.

\begin{tabular}{|c|c|c|}
\hline Muestras de Suelo & Cd(ppm) & $\operatorname{Cr}(\mathrm{ppm})$ \\
\hline $\mathrm{M}_{1}$ & 3,43 & 75,87 \\
\hline $\mathrm{M}_{2}$ & 0,50 & 10,54 \\
\hline $\mathrm{M}_{3}$ & 0,84 & 15,64 \\
\hline $\mathrm{M}_{4}$ & 0,94 & 14,55 \\
\hline $\mathrm{M}_{5}$ & 0,54 & 20,60 \\
\hline $\mathrm{M}_{6}$ & 1,24 & 25,84 \\
\hline $\mathrm{M}_{7}$ & 0,74 & 24,75 \\
\hline $\mathrm{M}_{8}$ & 1,24 & 35,70 \\
\hline $\mathrm{M}_{9}$ & 2,54 & 42,58 \\
\hline
\end{tabular}

Las variables a medir, fueron: rendimiento en base húmeda y seca, contenido de cadmio y cromo foliar y efecto de los tratamientos en el pH del suelo, después de la cosecha.

Análisis estadístico: Para las variables peso seco y peso fresco en las dos especies, se empleó el análisis de varianza, con el programa SAS y la comparación de medias, a través de la prueba de DMS. Los resultados foliares de cromo y de cadmio, para las dos especies vegetales bajo exploración, se estudiaron mediante el análisis de tendencia central (Calzada, 1964; Solanas et al.
2005) y, a partir del cambio porcentual en el contenido final de los tejidos foliares, comparando el testigo como base. Para el $\mathrm{pH}$ del suelo después de la cosecha, se adoptó, igualmente, el porcentaje de diferencia en relación al testigo.

Debido al elevado costo de los análisis químicos del material vegetal para la lechuga, se tomó una muestra promedia de cada uno de los 16 tratamientos y para ryegrass, una muestra promedia de cada uno de los tratamientos 1-3-4-6-7-9-1012-13-15 y 16 .

Tabla 2. Tratamientos utilizados en el bioensayo para lechuga y ryegrass.

\begin{tabular}{|c|c|c|}
\hline Tratamiento No. & Dosis kg/ha & Fuente remediadora \\
\hline 1 & 0 & Testigo \\
\hline 2 & 500 & \multirow{3}{*}{$\mathrm{CaSO}_{4}$} \\
\hline 3 & 1000 & \\
\hline 4 & 1500 & \\
\hline 5 & 2000 & \multirow{3}{*}{$\mathrm{CaCO}_{3}$} \\
\hline 6 & 4000 & \\
\hline 7 & 6000 & \\
\hline 8 & 300 & \multirow{3}{*}{$\mathrm{Ca}\left(\mathrm{H}_{2} \mathrm{PO}_{4}\right)_{2}$} \\
\hline 9 & 600 & \\
\hline 10 & 900 & \\
\hline 11 & 200 & \multirow{3}{*}{$\mathrm{FeSO}_{4}$} \\
\hline 12 & 400 & \\
\hline 13 & 600 & \\
\hline 14 & 2000 & \multirow{3}{*}{ Diatomácea activada } \\
\hline 15 & 4000 & \\
\hline 16 & 6000 & \\
\hline
\end{tabular}




\section{RESULTADOS Y DISCUSIÓN}

Rendimiento peso seco y húmedo ryegrass. En la tabla 3 , se observa el rendimiento en base húmeda y seca del pasto ryegrass. Con base en el análisis estadístico y en la prueba de comparación DMS (Diferencia Mínima Significativa) en peso fresco hubo efecto significativo de los tratamientos, con un coeficiente de determinación de 0,60 , indicando que el comportamiento de la variable dependió, en alto grado, del comportamiento de los tratamientos. Por su parte, el coeficiente de variación C.V. de 9,5 demuestra alta homogeneidad en las muestras analizadas y confiabilidad en los resultados obtenidos. Las dosis más altas de $\mathrm{CaCO}_{3}$ fueron superiores para rendimiento a los demás tratamientos, debido, posiblemente, a la reducción de la toxicidad del aluminio y al efecto en la reducción del cadmio y del plomo (Espinosa, 1994; Mejía, 1998). Por su parte, el tratamiento con $900 \mathrm{~kg} /$ ha de $\mathrm{Ca}\left(\mathrm{H}_{2} \mathrm{PO}_{4}\right)_{2}$ fue estadísticamente diferente al testigo, puesto que el aporte de fósforo mejoró el rendimiento del pasto. En cuanto al peso seco, en general, hubo la misma tendencia que la presentada en base húmeda para $\mathrm{CaCO}_{3}$. El incremento del rendimiento por aplicación de $\mathrm{CaCO}_{3}$ ocurrió, posiblemente, a la inactivación de elementos tóxicos y liberación de los nutrientes fijados en el suelo, que propician un mejor desarrollo radicular y, por tanto, de la planta (Bernal, 2008).

Peso seco y fresco en lechuga. De acuerdo con los datos consignados en la tabla 3 en peso fresco y seco, no hubo efecto significativo de los tratamientos; sin embargo, la aplicación de 300 o $900 \mathrm{~kg} / \mathrm{ha}$ de $\mathrm{Ca}\left(\mathrm{H}_{2} \mathrm{PO}_{4}\right)_{2}$ mostraron los rendimientos más elevados, dado, posiblemente, a una presencia adecuada de fósforo.

A diferencia del ryegrass, probablemente, porque la lechuga tiene buena tolerancia a cadmio y a cromo y a los niveles bajos de fósforo en el suelo. Para bajos niveles de fósforo y aluminio, la lechuga tiene buena tolerancia (Lora et al. 2006).

Efecto de los tratamientos en el contenido de cadmio y de cromo en ryegrass. En la tabla 4 aparece el efecto porcentual de algunos tratamientos en el contenido de cadmio y de cromo en el ryegrass. Para cadmio, el testigo presentó un contenido superior al nivel máximo aceptado por la Comunidad Europea,

Tabla 3. Efecto de los tratamientos en el rendimiento de ryegrass en base húmeda y seca.

\begin{tabular}{|c|c|c|}
\hline Tratamiento & Peso fresco (g) & Peso seco (g) \\
\hline $\mathrm{T}_{1}$ - Testigo & $58,01 \mathrm{DEF}$ & $16,47 \mathrm{CD}$ \\
\hline $\mathrm{T}_{2}-\mathrm{CaSO}_{4}-500 \mathrm{~kg} / \mathrm{ha}$ & $58,60 \mathrm{DEF}$ & $16,30 \mathrm{CD}$ \\
\hline $\mathrm{T}_{3}-\mathrm{CaSO}_{4}-1000 \mathrm{~kg} / \mathrm{ha}$ & $54,85 \mathrm{EF}$ & $16,33 \mathrm{CD}$ \\
\hline $\mathrm{T}_{4}-\mathrm{CaSO}_{4}-1500 \mathrm{~kg} / \mathrm{ha}$ & $49,87 \mathrm{~F}$ & $15,36 \mathrm{D}$ \\
\hline $\mathrm{T}_{5}-\mathrm{CaCO}_{3}-2000 \mathrm{~kg} / \mathrm{ha}$ & $58,45 \mathrm{DEF}$ & $17,49 \mathrm{CD}$ \\
\hline $\mathrm{T}_{6}-\mathrm{CaCO}_{3}-4000 \mathrm{~kg} / \mathrm{ha}$ & $71,14 \mathrm{AB}$ & $19,99 \mathrm{AB}$ \\
\hline $\mathrm{T}_{7}-\mathrm{CaCO}_{3}-6000 \mathrm{~kg} / \mathrm{ha}$ & $72,84 \mathrm{~A}$ & $20,17 \mathrm{~A}$ \\
\hline $\mathrm{T}_{8}-\mathrm{Ca}\left(\mathrm{H}_{2} \mathrm{PO}_{4}\right)_{2}-300 \mathrm{~kg} / \mathrm{ha}$ & $64,59 \mathrm{ABCD}$ & $17,14 \mathrm{CD}$ \\
\hline $\left.\mathrm{T}_{9}-\mathrm{Ca}_{2} \mathrm{H}_{2} \mathrm{PO}_{4}\right)_{2}-600 \mathrm{~kg} / \mathrm{ha}$ & $60,87 \mathrm{CDE}$ & $18,34 \mathrm{ABC}$ \\
\hline $\mathrm{T}_{10}-\mathrm{Ca}\left(\mathrm{H}_{2} \mathrm{PO}_{4}\right)_{2}-900 \mathrm{~kg} / \mathrm{ha}$ & $69,70 \mathrm{ABC}$ & $18,16 \mathrm{ABC}$ \\
\hline $\mathrm{T}_{11}-\mathrm{FeSO}_{4}-200 \mathrm{~kg} / \mathrm{ha}$ & $65,18 \mathrm{ABCD}$ & $18,83 \mathrm{ABC}$ \\
\hline $\mathrm{T}_{12}-\mathrm{FeSO}_{4}-400 \mathrm{~kg} / \mathrm{ha}$ & $58,39 \mathrm{DEF}$ & $17,58 \mathrm{BCD}$ \\
\hline $\mathrm{T}_{13}-\mathrm{FeSO}_{4}-600 \mathrm{~kg} / \mathrm{ha}$ & $58,16 \mathrm{DEF}$ & $18,10 \mathrm{ABC}$ \\
\hline $\mathrm{T}_{14}-{ }^{-} 2000 \mathrm{~kg} / \mathrm{ha}$ & $63,44 \mathrm{ABCDE}$ & $18,31 \mathrm{ABC}$ \\
\hline $\mathrm{T}_{15}-{ }_{4} \quad 4000 \mathrm{~kg} / \mathrm{ha}$ & $61,47 \mathrm{BCDE}$ & $17,69 \mathrm{BCD}$ \\
\hline $\mathrm{T}_{16}-{ }^{-} 6000 \mathrm{~kg} / \mathrm{ha}$ & $61,45 \mathrm{BCDE}$ & $16,49 \mathrm{CD}$ \\
\hline
\end{tabular}


Tabla 4. Efecto porcentual y análisis de tendencia central en el contenido de cadmio y de cromo en pasto ryegrass.

\begin{tabular}{|c|c|c|c|c|}
\hline Tratamientos (kg/ha) & $\begin{array}{c}\text { Cadmio } \\
(\mathrm{ppm})\end{array}$ & $\begin{array}{c}\text { \% diferencia en } \\
\text { relación al testigo }\end{array}$ & $\begin{array}{c}\text { Cromo } \\
(\mathrm{ppm})\end{array}$ & $\begin{array}{c}\text { \% diferencia en } \\
\text { relación al testigo }\end{array}$ \\
\hline Testigo & 0,60 & - & 2,49 & - \\
\hline $1000 \mathrm{~kg}-\mathrm{CaSO}_{4}$ & 0,49 & $-18,33$ & 2,40 & $-9,70$ \\
\hline $1500 \mathrm{~kg}-\mathrm{CaSO}_{4}$ & 0,60 & 0,00 & 2,19 & $-12,05$ \\
\hline $4000 \mathrm{~kg}-\mathrm{CaCO}_{3}$ & 0,30 & $-50,00$ & 1,49 & $-40,16$ \\
\hline $6000 \mathrm{~kg}-\mathrm{CaCO}_{3}$ & 0,20 & $-66,67$ & 1,70 & $-31,73$ \\
\hline $600 \mathrm{~kg}-\mathrm{Ca}\left(\mathrm{H}_{2} \mathrm{PO}_{4}\right)_{2}$ & 0,10 & $-83,33$ & 3,17 & 27,31 \\
\hline $900 \mathrm{~kg}-\mathrm{Ca}\left(\mathrm{H}_{2} \mathrm{PO}_{4}\right)_{2}$ & 0,40 & $-33,33$ & 5,26 & 111,24 \\
\hline $400 \mathrm{~kg}-\mathrm{FeSO}_{4}$ & 0,40 & $-33,33$ & 2,09 & $-16,06$ \\
\hline $600 \mathrm{~kg}-\mathrm{FeSO}_{4}$ & 0,40 & $-33,33$ & 2,73 & 9,64 \\
\hline $4000 \mathrm{~kg}-$ Diatomácea & 0,39 & $-35,00$ & 3,05 & 22,49 \\
\hline $6000 \mathrm{~kg}-$ Diatomácea & 0,40 & $-33,33$ & 2,75 & 10,44 \\
\hline
\end{tabular}

Análisis Tendencia Central:

\begin{tabular}{|c|c|c|}
\hline$\Sigma=$ & 4,28 & 35,62 \\
\hline Promedio= & 0,38909 & 3,23818 \\
\hline$S=$ & 0,15 & 2,07 \\
\hline C.V. $=$ & 38,71 & 63,93 \\
\hline
\end{tabular}

que es de 0,20ppm o de 0,23ppm, según Laredo y Cuesta (1987). Los tratamientos $6.000 \mathrm{~kg} / \mathrm{ha} \mathrm{CaCO}_{3}$ y $600 \mathrm{~kg} / \mathrm{ha}$ de $\mathrm{Ca}\left(\mathrm{H}_{2} \mathrm{PO}_{4}\right)_{2}$ disminuyeron el contenido a niveles no tóxico, posiblemente, por elevación del $\mathrm{pH}$ del suelo y por formación de fosfato de cadmio de baja solubilidad (Matamoros, 2003; Lora, 2007). En relación al contenido de cromo, los tratamiento con 4.000 y $6.000 \mathrm{~kg} / \mathrm{ha}$ de $\mathrm{CaCO}_{3}$ o con $400 \mathrm{~kg} / \mathrm{ha}$ de $\mathrm{FeSO}_{4}$ disminuyeron el contenido del elemento, siendo la aplicación de $4.000 \mathrm{~kg} / \mathrm{ha}$ de $\mathrm{CaCO}_{3}$ la dosis mas adecuada y, por tanto, es recomendable su evaluación, bajo condiciones de campo.

El análisis de tendencia central muestra diferencia en el contenido de cadmio, de acuerdo a los tratamientos, pues el C.V. es de 38,71 y 63,93, para los dos elementos, respectivamente. En relación al testigo para la mayoría de los tratamientos, la diferencia porcentual negativa es superior a
$20 \%$, indicando efecto de los tratamientos en la reducción de cadmio y de cromo (Calzada, 1964; Solanas et al. 2005).

Los datos de la tabla 4 advierten el problema de contaminación a niveles no permisibles de cadmio y de cromo en el suelo bajo estudio y la importancia de remediación, posteriormente, bajo condiciones de campo, para ryegrass, empleando algunas fuentes y dosis promisorias, halladas bajo condiciones de casa de malla.

Efecto de los tratamientos en el contenido de cadmio y de cromo en lechuga. En la tabla 5, se puede observar que todos los tratamientos redujeron la concentración de cadmio, mostrando un mayor efecto el carbonato de calcio. Con la aplicación de $6.000 \mathrm{~kg} / \mathrm{ha}$, el contenido del elemento se redujo, de 5,4ppm, en el testigo, a 0,60ppm; el porcentaje de 
diferencia fue de 89,03. Este comportamiento, probablemente, se debió a que el pH del suelo pasó de 4,80, en el testigo, a 6,63 y, en este grado de acidez, el cadmio reduce su solubilidad en el suelo y, por tanto, la absorción, por parte de la planta, se reduce. Por su parte, para el cromo en todos los tratamientos, se presentó disminución del elemento, siendo $400 \mathrm{~kg} / \mathrm{ha}$ de $\mathrm{FeSO}_{4}$ o $600 \mathrm{~kg} / \mathrm{ha}$ de $\mathrm{FeSO}_{4}$, los que mejor efecto presentaron, posiblemente, por competencia entre el cromo y el hierro, en los sitios de absorción, o por formación de sulfatos de cromo de baja solubilidad. Es importante considerar este comportamiento del sulfato ferroso para evaluarlo, bajo condiciones de campo. El análisis de tendencia central muestra efecto en los tratamientos en el contenido de cadmio y de cromo en la planta, puesto que el C.V. es de 39,75 y de 38,10 para los dos elementos, respectivamente. En relación al testigo, para la mayoría de los tratamientos, la diferencia porcentual fue superior al $20 \%$, indicando el efecto en los tratamientos en la reducción de cadmio y de cromo (Calzada, 1964; Solanas et al. 2005).

En la tabla 6, se reporta el efecto de los tratamientos en el $\mathrm{pH}$ del suelo después de la cosecha en ryegrass y lechuga, mostrando incremento superior al $20 \%$, respecto al testigo, para el $\mathrm{CaCO}_{3}$, y posible reducción de la disponibilidad de los elementos, para la planta. Debido a los elevados contenidos disponibles de cadmio y de cromo en el suelo bajo estudio es importante su remediación, para reducir el problema en lechuga y en pasto a nivel de campo, donde se produce la hortaliza y la gramínea. Se pueden emplear las fuentes y las dosis más promisorias, encontradas en la presente investigación.

Tabla 5. Efecto porcentual de los tratamientos en el contenido de cadmio y cromo en lechuga.

\begin{tabular}{|c|c|c|c|c|}
\hline Tratamientos (kg/ha) & Cadmio (ppm) & $\begin{array}{c}\text { \% diferencia en } \\
\text { relación al testigo }\end{array}$ & Cromo (ppm) & $\begin{array}{c}\text { \% diferencia en } \\
\text { relación al testigo }\end{array}$ \\
\hline Testigo & 5,40 & - & 6,23 & - \\
\hline $500 \mathrm{~kg}-\mathrm{CaSO}_{4}$ & 4,20 & $-22,30$ & 3,60 & $-42,54$ \\
\hline $1000 \mathrm{~kg}-\mathrm{CaSO}_{4}$ & 3,30 & $-38,85$ & 3,60 & $-42,38$ \\
\hline $1500 \mathrm{~kg}-\mathrm{CaSO}_{4}$ & 4,10 & $-24,72$ & 4,80 & $-22,95$ \\
\hline $2000 \mathrm{~kg}-\mathrm{CaCO}_{3}$ & 1,80 & $-66,54$ & 2,40 & $-61,48$ \\
\hline $4000 \mathrm{~kg}-\mathrm{CaCO}_{3}$ & 1,00 & $-81,41$ & 2,50 & $-59,87$ \\
\hline $6000 \mathrm{~kg}-\mathrm{CaCO}_{3}$ & 0,60 & $-89,03$ & 2,50 & $-60,19$ \\
\hline $300 \mathrm{~kg}-\mathrm{Ca}\left(\mathrm{H}_{2} \mathrm{PO}_{4}\right)_{2}$ & 3,30 & $-38,48$ & 2,70 & $-56,18$ \\
\hline $600 \mathrm{~kg}-\mathrm{Ca}\left(\mathrm{H}_{2} \mathrm{PO}_{4}\right)_{2}$ & 2,85 & $-47,03$ & 2,85 & $-54,25$ \\
\hline $900 \mathrm{~kg}-\mathrm{Ca}\left(\mathrm{H}_{2} \mathrm{PO}_{4}\right)_{2}$ & 2,90 & $-46,47$ & 2,90 & $-53,77$ \\
\hline $200 \mathrm{~kg}-\mathrm{FeSO}_{4}$ & 3,00 & $-44,24$ & 3,24 & $-47,99$ \\
\hline $400 \mathrm{~kg}-\mathrm{FeSO}_{4}$ & 3,98 & $-26,02$ & 1,90 & $-68,06$ \\
\hline $600 \mathrm{~kg}-\mathrm{FeSO}_{4}$ & 4,35 & $-19,14$ & 1,00 & $-83,47$ \\
\hline $2000 \mathrm{~kg}-\mathrm{Diatomácea}$ & 2,80 & $-47,58$ & 2,92 & $-53,13$ \\
\hline $4000 \mathrm{~kg}-\mathrm{Diatomácea}$ & 4,70 & $-12,64$ & 2,60 & $-58,75$ \\
\hline $6000 \mathrm{~kg}-\mathrm{Diatomácea}$ & 3,95 & $-27,77$ & 3,35 & $-46,23$ \\
\hline
\end{tabular}


Tabla 6. Efecto de los tratamientos en el pH del suelo después de la cosecha en ryegrass y lechuga.

\begin{tabular}{|c|c|c|c|c|c|c|}
\hline \multirow[b]{2}{*}{ Tratamientos } & \multirow[b]{2}{*}{$\mathrm{kg} / \mathrm{ha}$} & \multirow[b]{2}{*}{ Fuentes } & \multicolumn{2}{|r|}{ RYE GRASS } & \multicolumn{2}{|r|}{ LECHUGA } \\
\hline & & & $\mathrm{pH}$ final & $\begin{array}{c}\% \text { de diferencia en relación } \\
\text { al testigo }\end{array}$ & $\mathrm{pH}$ final & $\begin{array}{c}\% \text { de diferencia en relación } \\
\text { al testigo }\end{array}$ \\
\hline 1 & 0 & TESTIGO & 4,76 & Testigo & 4,8 & Testigo \\
\hline 2 & 500 & \multirow{3}{*}{$\mathrm{CaSO}_{4}$} & 4,77 & 0,21 & 4,83 & 0,63 \\
\hline 3 & 1000 & & 5,08 & 6,72 & 5,15 & 7,29 \\
\hline 4 & 1500 & & 4,89 & 2,73 & 5,05 & 5,21 \\
\hline 5 & 2000 & \multirow{3}{*}{$\mathrm{CaCO}_{3}$} & 5,49 & 15,34 & 5,78 & 20,42 \\
\hline 6 & 4000 & & 6,21 & 30,46 & 6,22 & 29,58 \\
\hline 7 & 6000 & & 6,46 & 35,71 & 6,63 & 38,13 \\
\hline 8 & 300 & \multirow{3}{*}{$\mathrm{Ca}\left(\mathrm{H}_{2} \mathrm{PO}_{4}\right)_{2}$} & 5,1 & 7,14 & 4,97 & 3,54 \\
\hline 9 & 600 & & 5,34 & 12,18 & 5,17 & 7,71 \\
\hline 10 & 900 & & 5,08 & 6,72 & 5,03 & 4,79 \\
\hline 11 & 200 & \multirow{3}{*}{$\mathrm{FeSO}_{4}$} & 5,18 & 8,82 & 5,25 & 9,38 \\
\hline 12 & 400 & & 5,16 & 8,4 & 5,2 & 8,33 \\
\hline 13 & 600 & & 5,18 & 8,82 & 5,36 & 11,67 \\
\hline 14 & 2000 & & 5,32 & 11,76 & 5,53 & 15,21 \\
\hline 15 & 4000 & Diatomácea & 5,02 & 5,46 & 5,35 & 11,46 \\
\hline 16 & 6000 & activada & 5,04 & 5,88 & 5,3 & 10,42 \\
\hline
\end{tabular}

Conflictos de intereses: El manuscrito fue preparado y revisado con la participación de todos los autores, quienes declaramos que no existe ningún conflicto de intereses que ponga en riesgo la validez de los resultados presentados. Financiación: Este estudio fue financiado por la Universidad de Ciencias Aplicadas y Ambientales U.D.C.A y los autores.

\section{BIBLIOGRAFÍA}

1. ACCIOLY, A.M.A ; SOARES, C.R.F.S.; SIQUEIRA, J.O. 2009. Silicato de cálcio como amenizante da toxidez de metais pesados em mudas de eucalipto. Pesq. Agropec. Bras. Brasilia. 44(2):180-188.

2. ÁLVAREZ, D.; CONTRERAS, S.; TRUJILLO, N.; FRÍAS, F.; OLALDE, V. 2006. Effects of tanneries on chemical and biological soil characteristics. Appl. Soil Ecol. 33:269-277.

3. ÁLVAREZ, F.; ENEDI, A.; HAMILTON, C.; MURAOKA, T.; TREVIZÁNN, A. 2008. Fitodisponibilidad de cadmio en suelo de diferente $\mathrm{pH}$ tratado con lodo albañal estimada por método isotópico y extracción química. Quim. Nova. 31(5):1020-1026.

4. ALFARO, R.; GARCÍA, E.; MONTENEGRO, O. 2002. Niveles de contaminación de mercurio, cadmio, arsénico y plomo en suelos de la Cuenca Baja del Río Bogotá. Rev. U.D.C.A Act. E Div. Cient. 4(2):66-71.

5. BADILLO, G.; POMARES, G. 1995. Metals. En: Curso Básico de toxicología ambiental. Ed.l Limusa. México. 311p.

6. BERNAL, F. 2008. Actualidad y tendencia de la fertilización de pastos. Soc. Col. Ciencia del Suelo. Bogotá. D.C. p.131-140.

7. BONOMELLI, C.; BONILLA, C; VALENZUELA, A. 2003. Efecto de la fertilización fosforada sobre el contenido de cadmio en cuatro suelos de Chile. Pesq. Aqrope. Bras. Brasilia. 38(10):1179-1186.

8. CALZADA, J. 1964. Métodos estadísticos para la investigación. Lima, Perú. 494p. 
9. CONCEICÃO, D.; JACQUIES, R.; BENTO, F.; SIMONETTI, A.; SELBACH, P.; CAMARGO, F. 2007. Redução de cromo hexavalente por bacterias isoladas de solos contaminados com cromo. Ciência Rural, Santa Maria. 37(6):1661-1667.

10. CUBEROS, E.; RODRÍGUEZ, A.; PRIETO, E. 2009. Niveles de cromo y alteraciones de salud en una población expuesta a las actividades de curtiembres en Bogotá, Colombia. Rev. Salud Pública. 11(2):278-289.

11. DATNOFF, L.E.; SNYDER, G.H.: KORNDORFER, G.H. 2001. Silicon in Agriculture. Elsevier Science, The Netherlands, 403p.

12. ESPINOSA, J. 1994. Acidez y encalado de los suelos. En: Fertilidad de Suelos Diagnóstico y Control. Sociedad Colombiana de la Ciencia del Suelo, Santa Fe de Bogotá. 345-403p.

13. ESTRADA, J. 2002. Pastos y Forrajes para el Trópico Colombiano. Ed. Universidad de Caldas. Manizales, Colombia, 293p.

14. GARCÍA, I.; DORRONSORO, C. 2005. Contaminación por metales pesados. En: Tecnología de suelos Universidad de Granada. Departamento de Edafología y Química Agrícola.

15. KEDE, M.L.F.M.; MOREIRA, J.C.; MAVROPOULOS, E.; ROSSI, A.M.; BERTOLINO, L.C.; PEREZ, D.V.; ROCHA, N.C.C. da. 2008. Estudo do comportamento do chumbo em latossolos brasileiros tratados com fosfatos: contribuições para a remediação de sítios contaminados. Quim. Nova. 31(3):379-584.

16. LAREDO, M.; CUESTA, A. 1987. Tabla de contenido nutricional de pastos y forrajes de Colombia. Segunda Edición. Bogotá, D.C. Instituto Colombiano Agropecuario. 80p.

17. LÓPEZ, M. 1976. Caracterización y clasificación de Suelos de la Serie Río Bogotá. Instituto Geográfico Agustín Codazzi. 110p.

18. LORA, R. 2007. Contaminación por micronutrientes y posibles soluciones. Rev. U.D.C.A. Act. E Div. Cient. 7(1):5-20.

19. LORA, R.; BEJARANO, M.; ORDÓÑEZ, R. 2002. Respuesta del arroz a la aplicación de silicio en Casanare, Colombia. Suelos Ecuatoriales, 32:7-13.
20. LORA, R. 1996. Recuperación de aguas contaminadas con selenio. En XIII Congreso Latino Americano de la Ciencia del Suelo. Aguas de Lindoia, Brasil. 12p.

21. LORA, R. 2001. El silicio en la agricultura con énfasis en la producción de arroz. En: Sociedad Colombiana de la Ciencia del Suelo. Los elementos secundarios y el silicio en la agricultura. p.125-135.

22. LORA, R.; PULIDO, I.; MÉNDEZ, A.; PEÑA, F. 2006. Efecto en la fertilización orgánica y mineral en el rendimiento de la lechuga (Lactuca sativa L.) en un suelo Typic Hapludalfs de mediana fertilidad. Rev. U.D.C.A Act. \& Div. Cientí. 10(1): 5-20.

23. MARSCHNER, H., 2003. Mineral nutition of higher plants. Function of micronutrients. Ed. Acad Press, San Diego, California, USA. 889p.

24. MATAMOROS, A. 2003. Suelos contaminados por metales traza. En: Sociedad Colombiana de la Ciencia del Suelo. Manejo Integral de la fertilidad del suelo. Ed. Guadalupe Ltda. Bogotá. p.23-30.

25. MEJÍA, L. 1998. Degradación de suelos y cultivos por contaminación con elementos traza y pesados de diversas fuentes de Colombia. En: Encuentro Nacional de Labranza de Conservación. Villavicencio, Biblioteca Germán Arciniegas. p.97-145.

26. MEJÍA, L. 2002. Contaminación de suelos, aguas y plantas en Colombia. Suelos Ecuatoriales. 32(2):247-295.

27. MÉNDEZ, L.; RODRÍGUEZ, L.; PALACIOS, S. 2000. Impacto del agua de riego con aguas contaminadas, evaluado por la presencia de metales pesados en suelos. Terra Latinoamericana. México. 18(4):277-288.

28. MUCHUWETI, M.; BIRKETT, J.; CHINYANGA, E.; ZVAUYA, R.; SCRIMSHA, M.; LESTER, J. 2006. Heavy metal content of vegetables irrigates with mixtures of waste water and sewage sludge in Zimbabwe: Implication for human health. Agr. Ecosystems and Environment. 112:41-48.

29. OROZCO, C.; PÉREZ, A.; GONZÁLEZ, M.; RODRÍGUEZ, F.; ALFAYATE, J. 2005. Contaminación Ambiental. Una visión desde la química. Thomson Editores, Madrid España. p.631-650.

30. OSORIO, J.; LOBO, A. 1977. Hortalizas, Manual de Asistencia Técnica ICA, División de Agronomía, Programa de Hortalizas. Bogotá, Colombia. p.515-526. 
31. PAIM, L.A.; CARVALHO, R.; ABREU, C.M.P.; GUERREIRO, M.C. 2006. Estudo dos efeitos do silício e do fósforo na redução da disponibilidade de metais pesados em área de mineração. Quím. Nova. 29(1):28-33.

32. PANDA, S.; CHOUDHURY, S. 2005. Chromium stress in plants. Braz. J. Plant Physiol. 17(1):95-102.

33. PETERSON, P.; GIRLING, C. 1981. Other Trace Metals. En: Leep, N.W. Ed. Effect of heavy metal pollution on plants. Vol 1: Effect of trace metals on plants function. Applied Science Publishers. London. 325p.
34. SIEBE, C. 1994. Heavy metal contamination of soils irrigated with untreated sewage effluent in Central Mexico. In: World Congress of Soils Science. Vol. 3 b. Mexico. p.98-399

35. SINHA, S.; KUMAR, S. 2009. Pseudomonas aeroginosa kucd1, a posible candidate for cadmium bioremediation. Braz. J. Microbiol.40:655-662.

36. SOLANAS, A.; SALAFRANCA, L.; FAUQUET, J.; NUÑEZ, I. 2005. Estadística descriptiva en Ciencias del Comportamiento. Universidad de Barcelona. España. 619p.

Recibido: Diciembre 1 de 2009

Aceptado: Noviembre 4 de 2010 